\section{K-Ras' Achilles' heel}

\author{
By Lev Osherovich, Senior Writer
}

Three research teams associated with Harvard Medical School have identified key proteins and pathways that K-Ras-driven tumors require for survival, providing points to disrupt the proliferation of cells that harbor drug-resistant mutations of the oncogene. These secondary targets include a bevy of kinases and other enzymes involved in cell division.

Many of these weak spots in the underlying wiring of K-Ras (KRAS)-driven tumors are already being pursued by cancer companies. Indeed, the new studies suggest that rather than being an unwanted complication, K-Ras activation may in fact increase the sensitivity of tumors to drugs that target these secondary pathways.

$\mathrm{K}$-Ras activation often occurs in non-small cell lung cancer (NSCLC), colon cancer and pancreatic cancer, and it leads to poor prognosis and response to therapy because of aggressive tumor growth. Previous studies had suggested that tumors with K-Ras mutations acquire other genetic alterations, ${ }^{1}$ but the importance of these other pathways for tumor survival was poorly understood.

"Most of the focus on targeting K-Ras-positive tumors has been the K-Ras pathway," said Jeffrey Settleman, professor of oncology at Massachusetts General Hospital. "These new studies point out that we can go beyond this pathway.”

"K-Ras is commonly mutated, and we can't touch that mutant allele with a drug" despite years of effort, said D. Gary Gilliland, SVP of oncology at Merck \& Co. Inc.

Robert Brown, SVP of Dicerna Pharmaceuticals Inc., said that KRas has proven an intractable target for small molecules because of its similarity to related enzymes and its complex intracellular dynamics.

"These papers are dramatic confirmation that small molecule programs against K-Ras have been blindsided by the complexities of the underlying biology," said Brown.

Dicerna is targeting K-Ras with dicer substrate RNA, a highly potent form of RNAi, and has a range of K-Ras knockdown formulations in preclinical development for a range of cancer indications.

The new studies "identify unique liabilities in cancer cells" and open a back door to inhibiting the growth of cells that carry otherwise drug-resistant K-Ras mutations, Gilliland added.

One study in Cell was led by Stephen Elledge, professor of genetics at Brigham and Women's Hospital. ${ }^{2}$ A second Cell study was from a team led by William Hahn, associate professor of medicine at the Dana-Farber Cancer Institute, and Gilliland, who at the time was professor of medicine at Brigham and Women's Hospital. ${ }^{3}$

The third study, in Cancer Cell, was led by Settleman. ${ }^{4}$

\section{Lethal interactions}

The three teams used genetic techniques in cell culture to uncover the adaptations that K-Ras-positive tumors undergo to compensate for changes caused by constitutive activation of the K-Ras pathway. Inactivation of these supporting proteins selectively killed K-Ras-positive tumors but not wild-type cells or tumors with normal K-Ras.

"We took a completely open-minded genetic approach to ask a simple question: what do K-Ras mutants need to survive," said Elledge.

His team screened a short hairpin RNA library covering 32,293 human genes and identified 77 that blocked the growth of K-Rasmutated tumor cells but not that of a similar tumor cell line with wild-type K-Ras.

Among these were a set of genes involved in mitosis and chromosome segregation, a process that goes into overdrive in tumors. For example, the researchers found that knocking down polo-like kinase 1 (PLK1; STPK13), anaphase promoting complex subunit 1 (ANAPC1) and ANAPC4 lowered the growth of K-Ras mutant cells but not wildtype cells.

The team observed similar effects with BI 2536, a small molecule PLK1 inhibitor from Boehringer Ingelheim GmbH that is in Phase II testing for cancer.

Other PLK1 inhibitors in the clinic include Boehringer Ingelheim's BI 6727, which is in Phase II trials for NSCLC and Phase I/IIa trials for acute myelogenous leukemia (AML); Onconova Therapeutics Inc.'s ON 01910. $\mathrm{Na}$, which is in Phase I trials for solid tumors; and GlaxoSmithKline plc's GSK461364, which is in Phase I for nonHodgkin's lymphoma (NHL).

Another company participating in the PLK1 game is Tekmira Pharmaceuticals Corp. Tekmira has a stable nucleic acid-lipid particle formulation of small interfering RNA targeting PLK1 in preclinical development for cancer.

"Elledge has done a really nice job of confirming that K-Ras mutant tumors appear to depend on various non-oncogenes," said Tekmira CSO Ian MacLachlan. "One of the reasons we chose PLK1 is that we found that we could inhibit tumor growth in a variety of cancers at relatively low doses. We came to analogous conclusions" as Elledge's team.

Elledge said that his team is now sorting through the list of other genes that are lethal to cells with K-Ras mutations to identify other potentially druggable targets. Besides PLK1, he noted that at least one other protein identified in the screen-NEDD8 activating enzyme (NAE) - has been explored as a cancer drug target. However, he added that the importance of K-Ras activity for the efficacy of compounds targeting these enzymes hasn't yet been tested in the clinic.

Millennium Pharmaceuticals Inc., a unit of Takeda Pharmaceutical Co. Ltd., is running Phase I studies of the NAE inhibitor MLN4924 in several cancer indications.

Elledge thinks that once the most druggable targets from his list are identified, a customized mixture of inhibitors of K-Ras tumor-supporting proteins could be tried in patients with K-Ras-positive tumors. 


\section{STK33 in the middle}

Hahn and Gilliland's team took a more focused approach than Elledge's group, screening a panel of tumor cell lines with a smaller shRNA library corresponding to 5,024 druggable cancer-related genes, including those that code for kinases and phosphatases.

The researchers ranked the resulting candidate shRNAs by strength of effect and breadth of action across multiple cell lines and converged on a previously uncharacterized protein-serine/threonine kinase 33 (STK33).

In 10 of 12 tumor lines bearing K-Ras mutations, shRNA knockdown of STK33 prevented growth compared with no treatment. STK33 knockdown did not significantly slow the growth of 12 tumor lines that had wild-type K-Ras.

"The majority of tumors that have mutant K-Ras depend on STK33 for survival," said Hahn. "You'd predict that STK33 would be a good target for a majority of K-Ras-mutant cancers."

"While there are a lot of proteins implicated in cancer, a lot of them are transcription factors and are thus undruggable," added Gilliland. "In our study, we identified targets that are more druggable. Identifying STK33 inhibitors should be the fun part."

Although little is known about the function of STK33, Hahn and Gilliland's teams found that it may inhibit another kinase that stimulates apoptosis. Thus, K-Ras-driven tumor cells may be more inclined to undergo cell death if STK33 is inactivated.

Gilliland told SciBX that "several pharmas are actively engaged" in screening for STK33 inhibitors since he first presented his team's findings at a conference several months ago. Gilliland would not disclose whether Merck was among them.

Similar to Elledge's view, Hahn expects that STK33 inhibitors would likely be part of a broader cocktail of therapeutics aimed at multiple targets. "You're going to have to do combination therapy even in K-Ras-positive tumors," he said.

Gilliland said his academic collaborators plan to validate STK33 as a target in animal models of K-Ras-driven cancers.

\section{Over odds}

Settleman's team took an entirely different approach to the same goal of identifying weak spots of K-Ras-positive tumors. His group compared the gene expression patterns of tumors that depend on K-Ras for growth with those of tumors that do not.

"We took a group of K-Ras mutant cell lines from lung and pancreas and asked what happens when you deplete K-Ras," said Settleman. "We assumed that most cells would require K-Ras similarly, but to our surprise we found that we could divide the cells into two groups-those that died when we knocked down K-Ras and those that didn't."

Using microarrays, the team created a transcriptional profile that distinguished the K-Ras-dependent cells and K-Ras-independent cells and then combed the profile for druggable proteins that were relatively overexpressed in K-Ras-requiring tumors.

High on the list were two kinases: syk tyrosine kinase (SYK) and macrophage stimulating 1 receptor, c-met-related tyrosine kinase (MST1R; RON). Both had previously been identified as cancer targets, though without a connection to K-Ras.

Settleman's team went on to show that K-Ras-dependent cells treated with shRNA against SYK or RON grew poorly compared with similarly treated K-Ras-independent cells. Likewise, K-Ras-requiring tumor lines were more sensitive to R406, a small molecule SYK inhibitor from Rigel Pharmaceuticals Inc., than K-Ras-independent tumors.

Settleman's findings are "consistent with SYK's known role in the survival of hematopoietic cells, such as B cells, mast cells and NK cells," said Donald Payan, Rigel's EVP and CSO. The Cancer Cell study "offers a rational approach for the treatment of certain K-Rasdependent cancers with the added benefit of potentially very useful biomarkers to stratify patients and monitor responses to therapies."

Rigel's tamatinib fosdium (R788), a prodrug of R406, is in Phase II trials to treat a range of solid tumors in patients who have failed first-line therapy.

Settleman thinks that the transcriptional profile created by his team could be used to stratify patients with K-Ras-dependent tumors who would be more responsive to SYK or RON inhibitors.

"In general, K-Ras-positive patients are refractory to treatment," he said. "But among these there might be patients with SYK- or RON-dependent tumors."

The researchers also found that the cell-surface receptor integrin $\beta_{6}$ (ITGB6) is overexpressed in K-Ras-dependent tumors. ITGB6 is the target of Stromedix Inc.'s STX-100, a mAb in Phase I testing for chronic allograft nephropathy. Stromedix in-licensed STX-100 from Biogen Idec Inc. in 2007 and researchers from both companies were coauthors on Settleman's paper.

Elledge noted that his team used a similar transcriptional profiling method and found that high levels of some of their identified genes boded poorly for patient survival.

There appears to be little overlap between the genes identified in Settleman's transcriptional analysis and the genes reported in the Cell studies. This could mean that K-Ras-positive tumors may use a variety of mechanisms to adapt to rampant growth and that these tumors may have a large number of potential weak spots, said Gilliland.

"Maybe there are a lot of proteins that we can think about targeting in this setting," agreed Settleman.

Dicerna's Brown added that combination therapies to hit multiple targets involved in K-Ras tumor survival should be easier with RNAi-based technologies than with small molecules. A formulation containing various small molecules with distinct pharmacodynamic properties makes optimization of drug delivery complicated, whereas combined delivery of dicer substrate RNAs does not have such pharmacodynamic impediments, he said.

None of the researchers has patented their findings.

Osherovich, L. SciBX 2(23); doi:10.1038/scibx.2009.929

Published online June 11, 2009

\section{REFERENCES}

1. Weinstein, I.B. \& Joe, A. Cancer Res. 68, 3077-3080 (2008)

2. Luo J. et al. Cell; published online May 29, 2009; doi:10.1016/j.cell.2009.05.006

Contact: Stephen J. Elledge, Brigham and Women's Hospital, Harvard Medical School, Boston, Mass. e-mail: selledge@genetics.med.harvard.edu

3. Scholl, C. et al. Cell; published online May 29, 2009; doi:10.1016/j.cell.2009.03.017

Contact: D. Gary Gilliland, Harvard University, Boston, Mass. e-mail: ggilliland@rics.bwh.harvard.edu 


\section{TARGETS \& MECHANISMS}

Contact: William C. Hahn, same affiliation as above e-mail: william_hahn@dfci.harvard.edu

4. Singh, A. et al. Cancer Cell; published online June 2, 2009; doi:10.1016/j.ccr.2009.03.022

Contact: Jeff Settleman, Harvard Medical School, Boston, Mass. e-mail: settleman@helix.mgh.harvard.edu

COMPANIES AND INSTITUTIONS MENTIONED Biogen Idec Inc. (NASDAQ:BIIB), Cambridge, Mass. Boehringer Ingelheim GmbH, Ingelheim, Germany Brigham and Women's Hospital, Boston, Mass. Dana-Farber Cancer Institute, Boston, Mass.
Dicerna Pharmaceuticals Inc., Boston, Mass.

GlaxoSmithKline plc (LSE:GSK; NYSE:GSK), London, U.K.

Harvard Medical School, Boston, Mass.

Massachusetts General Hospital, Boston, Mass.

Merck \& Co. Inc. (NYSE:MRK), Whitehouse Station, N.J.

Millennium Pharmaceuticals Inc. (NASDAQ:MLNM), Cambridge, Mass.

Onconova Therapeutics Inc., Princeton, N.J.

Rigel Pharmaceuticals Inc. (NASDAQ:RIGL), South San Francisco, Calif.

Stromedix Inc., Cambridge, Mass.

Takeda Pharmaceutical Co. Ltd. (Tokyo:4502), Osaka, Japan

Tekmira Pharmaceuticals Corp. (TSX:TKM), Burnaby, British Columbia, Canada 